\title{
Antimicrobial Properties of Copper and d-Orbital Capture
}

\section{Peter Love}

Chemistry Department, University of Connecticut at Stamford, I University Place, Stamford, Connecticut, USA

Correspondence to: Peter Love, peter.love@uconn.edu

Keywords: Antimicrobial Properties of Copper, d-Orbital Capture, Ultraviolet Radiation Frequency, Cuprate Superconductivity

Received: October 19, $2020 \quad$ Accepted: February 22, $2021 \quad$ Published: February 25, 2021

Copyright $\odot 2021$ by author(s) and Scientific Research Publishing Inc.

This work is licensed under the Creative Commons Attribution International License (CC BY 4.0).

http://creativecommons.org/licenses/by/4.0/

\section{(c) (i) Open Access}

\section{ABSTRACT}

The purpose of this note is to stimulate interest in measuring and characterizing the emitted ultraviolet frequencies in antimicrobial copper materials. Antimicrobial sanitizing materials are urgently needed to limit the spread of COVID-19 virus. In the current pandemic, ultraviolet radiation is often used for sterilization. It is shown that $3 \mathrm{~d}$-orbital capture in copper can result in radiation generated by copper materials. Since ultraviolet radiation is known to be effective in antimicrobial sterilization, it is logical to assume that the radiation formed by copper occurs in the ultraviolet region. Electron transitions in $3 \mathrm{~d}$-orbital capture are expected to occur in this region. A description of the $3 \mathrm{~d}$-orbital capture process, and the origin of the associated frequency, is given. It is shown that for Group 1B elements the strength of electron affinity in the d-orbital capture process increases with increasing Periodic Table period number, $n$. This is the opposite of other electron affinity properties for atoms that decrease wth an increase in $\mathbf{n}$. A brief discussion of the relationship of d-orbital capture to the chemical inertness of gold is given. The same type of d-orbital capture process that occurs in antimicrobial copper occurs in high temperature superconducting cuprates.

\section{INTRODUCTION}

There is an urgent need to develop effective antimicrobial systems and materials to combat the spread of COVID-19 virus. This is being done in many ways to protect people, public facilities, businesses, and the home environment. Sterilization by use of ultraviolet, UV, radiation is well known [1]. This method is radiation time dependent, and has effective ranges on the order of meters. Solid materials that have the ability to permanently kill viruses on physical contact have been developed. These materials in principle are time independent. However, they have limited effective ranges due to the necessity of contact between the antimicrobial device and microbe sources. Reportedly the US Environmental Protection Agency listed 200 antimicrobial products in March, 2020. By mid-November, 2020 the list was 508 [2].

Our concern here is with solid materials that have continuous, and preferably permanent, antimi- 
crobial activity. A number of elemental metals are known to be effective [3]. One of the most commonly discussed metals is copper. Since UV radiation is known to be very effective for antimicrobial sterilization it is logical to assume that the antimicrobial properties of copper are due to UV radiation emitted by this element. The purpose here is to show the electronic basis for the effectiveness of copper and copper derivatives. It is unrealistic to believe that all antimicrobial materials have a common effective mechanism.

\section{COPPER AS AN ULTRAVIOLET RADIATION SOURCE}

Copper is one of the IB group of elements, copper, silver and gold, in the Periodic Table. The A-B group convention as discussed by Lopper [4] is used herein. Elements in a vertical column in the Periodic Table constitute a group. Positions of groups of elements with characteristic chemical reactivities in the Periodic Table are obscured when the IUPAC 1 - 18 group designation is used.

(In the A-B group convention the A group consists of the representative elements where the group number indicates the number of valence electrons in an atom. The B group consists of transition elements where d-orbitals first occur in the electron filling order. It is the process of d-orbital capture that causes the next to last column of the B group elements to be named the IB group).

\subsection{3 d-Orbital Capture in Copper}

The electron configuration of the element copper with respect to electron filling order should be,

$$
\mathrm{Cu}:[\mathrm{Ar}]^{18} 4 \mathrm{~s}^{2} 3 \mathrm{~d}^{9} \text {. }
$$

(Equation (1) is a short hand notation to represent electron configurations of elements in the Periodic Table. The term, $[\mathrm{Ar}]^{18}$, i.e., brackets enclosing an element with an upper right hand number indicates that all 18 electrons up to, and including those of the element, are filled. The term, $4 \mathrm{~s}^{2} 3 \mathrm{~d}^{9}$, represents the successive filling order of orbitals starting in the $4^{\text {th }}$ period, i.e., $\mathrm{n}=4$, as one scans across a period from left to right in the Periodic Table).

Since copper is in Group IB, with one electron in the valence shell, the stable electron configuration in terms of electron filling order is,

$$
\mathrm{Cu}:[\mathrm{Ar}]^{18} 4 \mathrm{~s}^{1} 3 \mathrm{~d}^{10} \text {. }
$$

The stable valence shell is expected to be, $4 \mathrm{~s}^{2}$, whereas it actually is, $4 \mathrm{~s}^{1}$. This results in a facile equilibrium, or resonance, of the itinerant electron as shown in Equation (3).

$$
4 \mathrm{~s}^{2}+3 \mathrm{~d}^{9} \rightleftarrows 4 \mathrm{~s}^{1}+3 \mathrm{~d}^{10} \text {. }
$$

The phrase, " $3 \mathrm{~d}$-orbital capture" refers to the electromeric shift process whereby an itinerant electron is reversibly abstracted from the $4 \mathrm{~s}^{2}$ valence shell of copper, and completes filling of the $3 \mathrm{~d}$-subshell. This is indicated by Equation (3). This dynamic equilibrium results in emitted radiation of frequency, $v$. The emitted radiation is the result of changes in the atomic orbitals, $4 \mathrm{~s}^{2}$, and $3 \mathrm{~d}^{9}$. The copper atom for Equation (3) remains electrically neutral. These orbitals have substantial orbital overlap since they are in close proximity. Therefore, the itinerant electron moves only a very short distance. Consequently the emitted radiation is of low intensity. This frequency is expected to occur in the ultraviolet region.

Resonance frequency equations equivalent to Equation (3) can be written for species such as $\mathrm{Cu}^{!+}$. The primary requirement for such equations is that the copper species has a vacant d-orbital. The frequency and intensity of radiation will, of course, vary with the copper species considered.

For silver a " $4 \mathrm{~d}$-orbital capture" equation equivalent to Equation (3) can be written.

$$
5 \mathrm{~s}^{2}+4 \mathrm{~d}^{9} \rightleftarrows 5 \mathrm{~s}^{1}+4 \mathrm{~d}^{10} .
$$

Electron affinity for d-orbital completion increases with increasing period number, $\mathrm{n}$. This is shown to be true in Section 2.2. Here $\mathrm{n}$ is a period, i.e., a horizonal row, in the Periodic Table. Therefore, the equilibrium in Equation (4) is expected to be shifted more to the right relative to that of Equation (3). The 
equilibrium in Equation (3) is a lower energy process than that in Equation (4). Therefore, the radiation frequency for copper is expected to be lower than that for silver.

\subsection{Effects of d-Orbital Capture on Atom Properties}

The key to understanding the chemical and physical properties of Group IB elements is to recognize the strong effect of d-orbital capture on electron affinity in these elements. This effect is strong enough to uncouple the paired spins of a Lewis pair in a valence shell. This obviously is true since the stable valence shell configurations are $\mathrm{ns}^{1}$. It is also strong enough to prevent oxidation at room temperature of the $6 \mathrm{~s}^{1}$ electron in gold. This is discussed in Section 2.3.

Not only is the d-orbital capture effect strong, it is the opposite of what is expected in terms of electrostatic attraction of valence shell electrons by the positively charged nucleus of an atom. For Group IA elements where valence shell electrons are removed in oxidation, as the period, $\mathrm{n}$, increases, the Pauling electronegativity, $\chi$ [5], steadily decreases. For $\mathrm{n}$ increasing: $2 \rightarrow 6(\mathrm{Li} \rightarrow \mathrm{Cs}), \chi$ decreases: $0.98 \rightarrow 0.79$. This is as expected. For Group VIIA elements where electrons are captured in reduction, for $\mathrm{n}$ increasing: $2 \rightarrow 6$ $(\mathrm{F} \rightarrow \mathrm{At}), \chi$ decreases: $3.98 \rightarrow 2.2$. The electronegativity steadily decreases as is expected.

For the Group IB elements where d-orbital capture is operative, for $\mathrm{n}$ increasing:

$4 \rightarrow 6(\mathrm{Cu} \rightarrow \mathrm{Au}), \chi$ increases: $1.90 \rightarrow 2.54$. This is the opposite of what is expected in terms of orbital electron - nuclear charge attraction. This can only be explained by noting the strong electron affinity that occurs in d-orbital capture.

The same effect of d-orbital capture is shown for atomic electron affinity, $\beta[6]$.

The electron affinity increases with increasing $\mathrm{n}$. This is shown in Table 1, Column 4.

From Table 1, Column 3, it is clear that the ease of oxidation decreases rapidly as the period, n, increases. For copper two electrons can be ionized to form a $2+$ oxidation state at $25^{\circ} \mathrm{C}$. For silver only a $1+$ oxidation state is found. Gold is chemically inert to oxidation at $25^{\circ} \mathrm{C}$. Therefore, the maximum ion oxidation number is zero for this element. This is expected to be primarily due to the increase in nuclear charge with increasing $\mathrm{n}$.

The d-orbital capture effect is also shown by electromotive force, EMF, $\mathrm{E}^{\circ}$, values, [7]. This is shown in Table 1, Column 5. As $\mathrm{n}$ increases the value of $\mathrm{E}^{\circ}$ increases.

Table 1. Chemical and Physical Properties of Group I B Elements.

\begin{tabular}{cccccc}
\hline \multirow{2}{*}{ Element } & $\begin{array}{c}\text { Per. Table } \\
\text { Period, } \mathrm{n}\end{array}$ & $\begin{array}{c}\text { Max. Ion Oxid. } \\
\text { Number }^{\mathrm{a}}\end{array}$ & $\begin{array}{c}\text { Electron Affinity } \\
\beta(\mathrm{eV})\end{array}$ & $\begin{array}{c}\text { Oxid. Pot. }^{\mathrm{b}} \\
\mathrm{E}^{\circ}(\mathrm{V})\end{array}$ & $\begin{array}{c}\text { Conduct. } \\
\sigma\left(\mathrm{S} \cdot \mathrm{m}^{-1}\right)\end{array}$ \\
\hline $\mathrm{Cu}$ & 4 & $2+$ & 1.226 & -0.521 & $5.90 \times 10^{7}$ \\
$\mathrm{Ag}$ & 5 & $1+$ & 1.303 & -0.799 & $6.30 \times 10^{7}$ \\
$\mathrm{Au}$ & 6 & 0 & 2.309 & -1.68 & $4.10 \times 10^{7}$ \\
\hline
\end{tabular}

a. At $25^{\circ}$ C. b. Electromotive Force, EMF, for 1 electron oxidation.

\subsection{Properties of Gold}

Since gold is a Group IB element some relevant comments are given here. As stated in Section 2.2 gold is chemically inert to oxidation at room temperature. Therefore, as far as chemical oxidation is concerned, a d-orbital capture frequency is not expected. However, the properties of gold are anomalous. It is the high mobility of the single valence shell electron of Group IB elements that results in the highest electrical conductivity, $\sigma$, of these elements in the Periodic Table. The conductivities of the Group IB elements are given in Table 1, Column 6 [5].

The very high conductivity, and $6 \mathrm{~s}^{1}$ valence shell occupancy, indicate that virtual d-orbital capture is still in effect. Ostensibly this process is simply the result of orbital overlap of one 6 s-electron and one $5 \mathrm{~d}$ - 
electron. If this were true the remaining $6 \mathrm{~s}$ electron should be available for ionization. However, as previously stated, gold is remarkedly inert to ionization at room temperature.

\section{DISCUSSION}

Explanation of the origin of antimicrobial activity of copper as being due to ultraviolet radiation emitted by this element is given in Sections 2.1 and 2.2. The antimicrobial properties of copper and copper related materials have been described by Selvamni, et al. [3]. These authors mention the presence of $\mathrm{CuO}$ and $\mathrm{Cu}_{2} \mathrm{O}$ in "laser texturing" samples as being responsible for enhanced antimicrobial activity as compared to that of pure copper [3]. Corning and PPG Industries are marketing a series of glass and paint encapsulated antimicrobial products that they claim have $\mathrm{Cu}^{1+}$ as the effective antimicrobial agent [2].

Measurement of the frequency of radiated energy in copper is important to verify the statement made herein that the antimicrobial activity of copper is the result of UV radiation formed by a 3 d-orbital capture equilibrium. Measurement of frequencies in related copper species that have antimicrobial activity will be useful to identify the source of antimicrobial activity in these species. It is of interest to determine if such frequencies exist in other types of antimicrobial materials. By such measurements, it may be possible to determine if there is a common mechanism for antimicrobial activity.

\section{RELATIONSHIP OF 3 d-ORBITAL CAPTURE IN COPPER TO CUPRATE SUPERCONDUCTIVITY}

Determination of this frequency in copper is also important academically. This should help to verify the postulate of $3 \mathrm{~d}$-orbital capture as being the cause of cuprate high temperature superconductivity [8]. If this postulate is true, irradiation of the superconductors at the appropriate frequency, $\mathrm{v}$, is expected to affect some of the properties of these materials. High temperature cuprate superconductivity requires a sustained source of mobile electrons to populate the superconducting state. The logical source of this mobile electron density is the equilibrium shown in Equation (3). It is the $3 \mathrm{~d}$-orbital capture resonant equilibrium that leads to superconductivity [8]. Since $3 \mathrm{~d}$-orbital capture equilibrium occurs in superconducting cuprate materials, there will be an associated characteristic frequency, $v$. It is possible that some of these materials above the transition temperature, $\mathrm{T}_{c}$, may also exhibit antimicrobial activity.

The high temperature superconductivity paper [8] complements this note. This note shows how UV radiation is generated in copper by $3 \mathrm{~d}$-orbital capture. In the superconductivity paper it is shown that itinerant electrons formed by $3 \mathrm{~d}$-orbital capture actually exist. This is the only way that itinerant electrons as superconducting bosons would form.

\section{A Suggested Experiment for an Enterprising Researcher, or a Graduate Student}

Rather than do a random search for the UV resonant frequency in copper it might be better to do a systematic search on a superconducting cuprate. It was just stated that there should be some anomaly in a cuprate above, or near, the superconducting transition temperature, $T_{c}$. If this is true, by irradiating an appropriately prepared sample and scanning the UV frequency spectrum with this irradiation, at some frequency an anomaly should be observed. This frequency for a cuprate is expected to be different from that for copper metal. However, it should be close enough to soon find the frequency in copper. It is, of course, possible that UV radiation might have some other effect, or effects, other than exciting the sample at the UV resonant frequency. Even so, this would be an interesting observation.

\section{SUMMARY}

The facts that copper has antimicrobial properties and that ultraviolet radiation is very effective for COVID-19 sanitizing strongly suggest that copper produces radiation in the ultraviolet region. Measurement of the UV frequency in copper is a first step to systematically characterize solid antimicrobial materials. Ultraviolet frequency measurements on known antimicrobial copper materials should be possible in 
university, or government, laboratories that have appropriate UV spectrometric instrumentation.

With cooperation of UV laboratories in different countries it would be good to make qualitative, and possibly quantitative, comparisons of the efficacy of antimicrobial activity for different materials. This could be correlated through the World Health Organization. The need to find efficient and practical antimicrobial sanitizers is very great.

\section{AGREE TO CONDITIONS}

I agree to conditions required by the Publisher for publication of this manuscript.

\section{CONFLICTS OF INTEREST}

The author declares no conflicts of interest regarding the publication of this paper.

\section{REFERENCES}

1. Kowalski, W. (2009) Ultraviolet Germicidal Irradiation Handbook. Springer, New York, 1-13. https://doi.org/10.1007/978-3-642-01999-9

2. Bettenhausen, C.A. (2020) A Paint to Kill COVID-19. Chemical and Engineering News, 98, 19. https://doi.org/10.47287/cen-09846-feature2

3. Selvamani, V., Zareei, A., Elkashif, A., Maruthamuthu, M.K., Chittiboyina, S., Delisi, D., et al. (2020) Hierachical Micro/Mesoporous Copper Structure with Enhanced Antimicrobial Property via Laser Surface Texturing. Advanced Materials Interfaces, 7, 1-11. https://doi.org/10.1002/admi.201901890

4. Lopper, M.E. (2019) A Digital Periodic Table That Instructors Can Use in the Classroom to Highlight Elements and Illustrate Periodic Trends. Journal of Chemical Education, 96, 387-389.

https://doi.org/10.1021/acs.jchemed.8b00616

5. Sargent-Welch (1979) Table of Periodic Properties of the Elements. Sargent-Welch Scientific, Skokie.

6. Dean, J.A. (1979) Lange's Handbook of Chemistry. 12th Edition, McGraw-Hill, New York, 3-10.

7. Masterton, W.L. and Hurley, C.N. (2004) Chemistry Principles and Reactions. 5th Edition, Brooks/Cole, Belmont, 481 .

8. Love, P. (2019) Orbital Approach to High Temperature Superonductivity. Natural Science, 11, 1-7. https://doi.org/10.4236/ns.2019.111001 